Paper

\title{
HEVC-based Light-field Coding using Basis Images and Frame Reordering
}

\author{
Kota Imaeda $^{\dagger}, \quad$ Keita Takahashi (member) ${ }^{\dagger}, \quad$ Toshiaki Fujii (member) ${ }^{\dagger}$, \\ Yukihiro Bandoh (member) ${ }^{\dagger \dagger}$, Seishi Takamura (member) ${ }^{\dagger \dagger}$, Hideaki Kimata (member ${ }^{\dagger \dagger}$
}

\begin{abstract}
An efficient coding method for light fields (LFs) is presented. The method is based on a sophisticated video coding standard called High Efficiency Video Coding (HEVC), but dose not directly encode the LF images using the HEVC codec. Instead, the LF images are first transformed into a set of smaller number of images, called basis images, to remove the redundancies among the images. The basis images are then reordered to produce a temporally smooth video sequence, which is finally encoded using the HEVC codec. In the decoding process, the decoded frames are inversely transformed into the original LF. The first and final transformations are modeled using neural networks and optimized for the target LF. The frame reordering is formulated as a traveling salesman problem (TSP) and solved using a greedy method. The experimental results show that our method can achieve better rate-distortion performance than other HEVC-based light-field coding methods.
\end{abstract}

Key words: Light field, HEVC, compression

\section{Introduction}

Light fields (LFs) $[1,2]$ are used to describe $3 \mathrm{D}$ visual information as light rays in 3D space and are utilized in various applications such as depth estimation [3], refocusing, and 3D display [4]. LF information can now be captured with light-field cameras [5,6], such as Lytro cameras. An LF is usually represented as a set of multiview images, i.e., dozens of images aligned on a $2 \mathrm{D}$ squared grid, viewing the same objects from slightly different angles. In its original format, such an LF has a significant amount of data, so developing efficient compression schemes is an important research objective, as evidenced by the many reported studies [7-14]. Among the reported schemes, we focused on a coding scheme where the given LF (multi-view) images are regarded as a sequence of temporal video frames and are encoded using the latest video coding standard, the High Efficiency Video Coding (HEVC) $[15,16]$.

To enhance the rate-distortion performance of this scheme, we focused on two important points. The first point is the issue of how to determine the frame order of the LF images. The frame order affects the temporal smoothness of the generated video sequence, which in

Received August 29, 2020; Revised November 4, 2020; Accepted November 16, 2020

$\dagger$ Nagoya University, Graduate School of Engineering

(Furo-cho, Chikusa-ku, Nagoya-shi, Aichi-ken, 464-8603, Japan) $\dagger \dagger$ NTT Media Inteligence Laboratories

(1-1 Hikarinooka, Yokosuka-shi, Kanagawa-ken, 239-0847, Japan) turn affects rate-distortion performance. Various frame orders in addition to the straightforward raster order have been tested [12-14]. Furthermore, to enhance performance, adaption of the frame order to the specific LF rather than using a fixed order for all LFs has been proposed $[17,18]$. The second point is the limited ability of video codecs to handle redundancy among multiple images. Although inter-frame prediction schemes have become increasingly sophisticated, the images are still treated as individual frames. Instead of encoding all the images, it would be more efficient to encode only a limited number of images (we call them "basis images") in which the information in the original images is "condensed" in some way. The result would be a set of basis images that are video codec friendly; i.e., they would be efficiently encoded as a natural video sequence.

Keeping these points in mind, we develop an HEVCbased LF coding method that uses basis images and optimized frame reordering. In this method, the set of original LF images is first transformed into a set of basis images, which are then reordered to create a temporally smooth video sequence that is finally encoded using the HEVC codec. In the decoding process, the frames decoded from the HEVC bit-stream are inversely transformed into the original light-field images. The first and final transformations are modeled using neural networks and optimized for the target LF. The frame reordering is formulated as a traveling salesman problem (TSP) and solved using a greedy method developed 


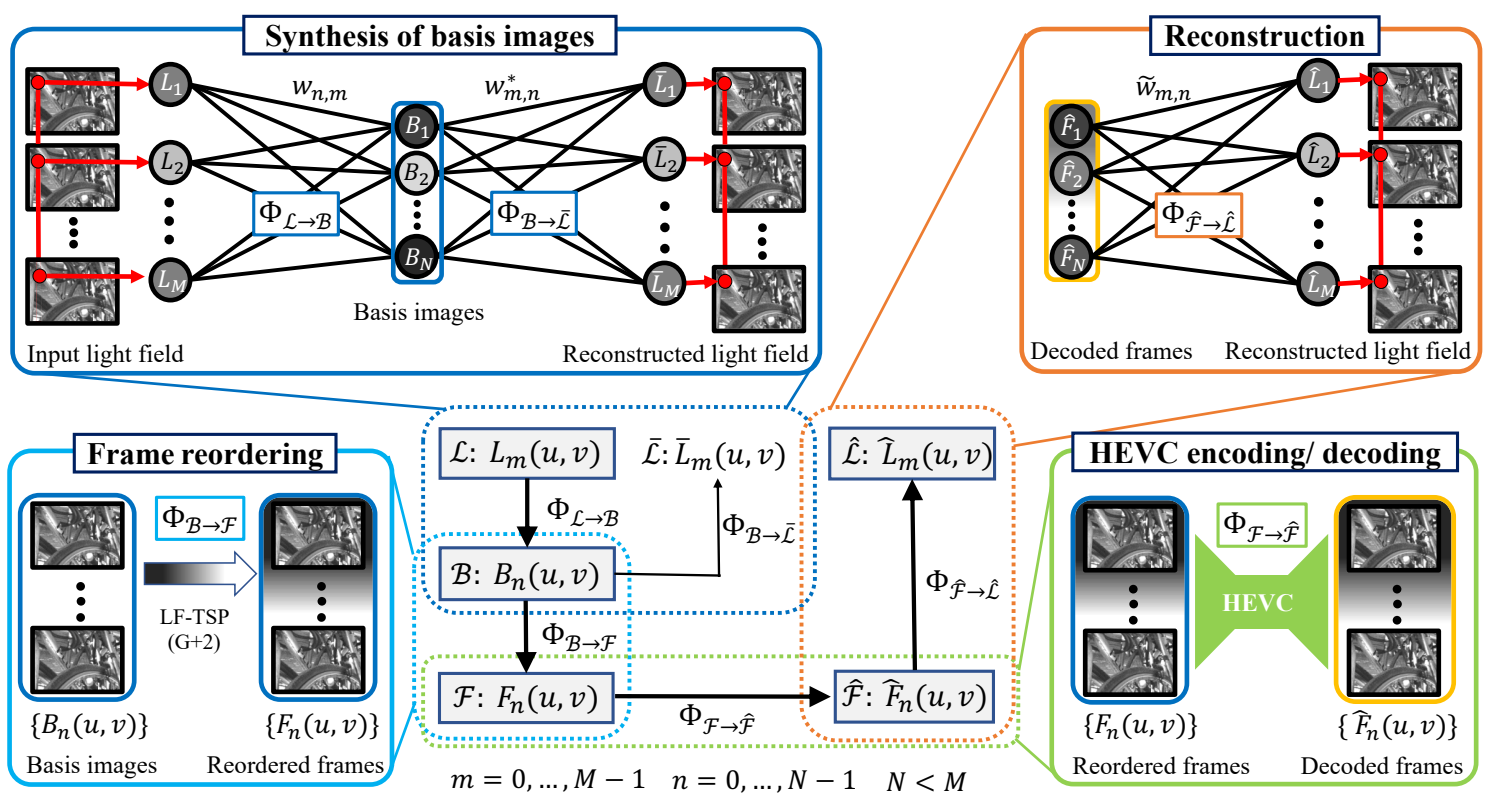

Fig. 1 Overview of our method. Input LF images $\mathcal{L}$ are first transformed into basis images $\mathcal{B}$, which in turn are reordered to produce a sequence of frames $\mathcal{F}$ and encoded using HEVC. The decoded frames $\hat{\mathcal{F}}$ are inversely transformed to reconstruct the LF images $\hat{\mathcal{L}}$.

by Imaeda et al. [18], resulting in a content-dependent frame order. The parameters for the final transformation are transmitted to the receiver along with the HEVC-encoded bit-stream. The experimental results show that this method achieves better rate-distortion performance than other HEVC-based LF coding methods $[12-14,18]$.

Reflecting the recent explosive prevalence of deep neural networks, learning-based video coding methods [19-22] have attracted much interest. However, these methods are still struggling to outperform the sophisticated video codecs. For example, although the method developed by Habibian et al. [22] has performance comparable to that of HEVC, their anchor, referred to as "HEVC", was actually the FFmpeg with the default configuration, which has a performance substantially inferior to that of the well-tuned HEVC reference software, HM [23], maintained by the Joint Collaborative Team on Video Coding (JCT-VC). Meanwhile, our method can incorporate any HEVC codecs. Thus, our method benefits from both the excellent ratedistortion performance of well-designed HEVC codec and the data-driven optimization brought by neural networks; in our method, we use a highly sophisticated video codec for encoding the basis images, and neural networks for optimizing the transformations between the LF images and basis images.

\section{Proposed method}

\subsection{Overview}

An overview of our method is shown in Fig. 1. The LF to be compressed, consisting of $M$ multi-view images, is denoted as $\mathcal{L}:=\left\{L_{m}(u, v) \mid m=0, \ldots, M-1\right\}$, where $m$ is the viewpoint index, and $(u, v)$ specifies a pixel. Instead of directly encoding $\mathcal{L}$ using an HEVC codec, two steps are performed in advance to achieve better rate-distortion performance. First, $\mathcal{L}$ is transformed into a set of smaller number of images, denoted as $\mathcal{B}:=\left\{B_{n}(u, v) \mid n=0, \ldots, N-1\right\}$, where $N<M$. This reduces the intrinsic redundancy among the images. We call $B_{n}(u, v)$ a basis image. We approximately span the target LF with the set of basis images $(\mathcal{B})$. The basis images in $\mathcal{B}$ are then reordered to obtain a sequence of frames, described as $\mathcal{F}:=\left\{F_{n}(u, v) \mid n=0, \ldots, N-1\right\}$, which is then compressed using the HEVC codec. The frame order is optimized to boost the rate-distortion performance of the codec. In the decoding process, a set of decoded frames described as $\hat{\mathcal{F}}:=\left\{\hat{F}_{n}(u, v) \mid n=0, \ldots, N-1\right\}$ is first obtained, from which the target LF, denoted as $\hat{\mathcal{L}}:=\left\{\hat{L}_{m}(u, v) \mid m=0, \ldots, M-1\right\}$, is reconstructed.

The optimal LF coding is achieved by minimizing the squared error $\left(\|\mathcal{L}-\hat{\mathcal{L}}\|^{2}\right)$ under a fixed bit-rate. This can be rewritten as

$$
\min \left\|\mathcal{L}-\Phi_{\hat{\mathcal{F}} \rightarrow \hat{\mathcal{L}}} \circ \Phi_{\mathcal{F} \rightarrow \hat{\mathcal{F}}} \circ \Phi_{\mathcal{B} \rightarrow \mathcal{F}} \circ \Phi_{\mathcal{L} \rightarrow \mathcal{B}}(\mathcal{L})\right\|^{2},
$$




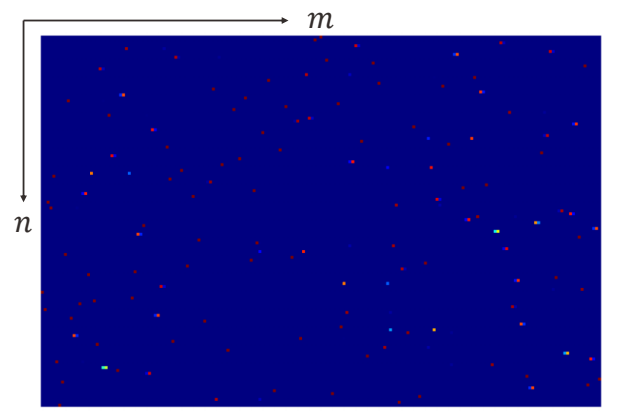

(a) Weights for $\Phi_{\mathcal{L} \rightarrow \mathcal{B}}\left(w_{n, m}\right)$

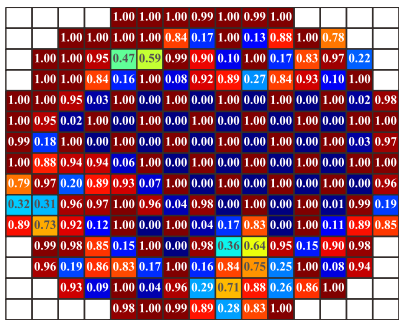

(b) Contrib. map

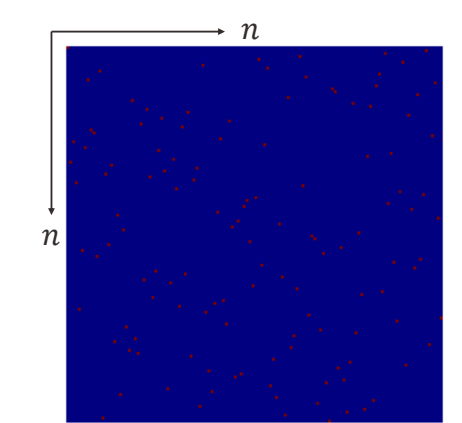

(c) Weights for $\Phi_{\mathcal{B} \rightarrow \mathcal{F}}$

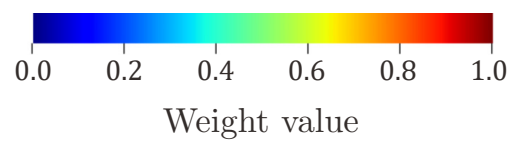

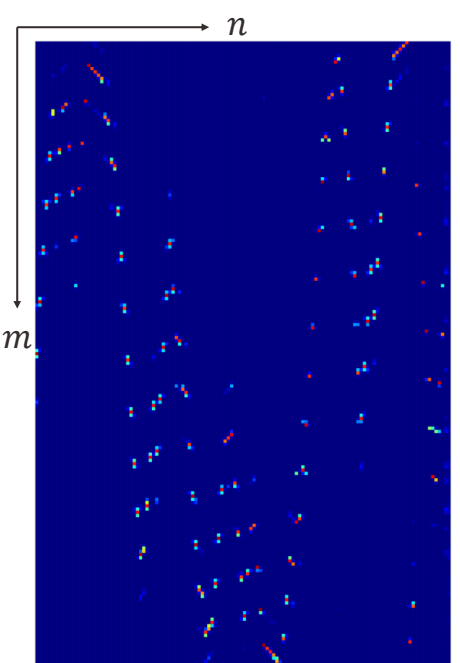

(d) Weights for $\Phi_{\hat{\mathcal{F}} \rightarrow \hat{\mathcal{L}}}\left(\tilde{w}_{m, n}\right)$

Fig. 2 Example of mapping operators for Bikes $\operatorname{LF}((M, N)=(193,128))$.

where the transformation processes are denoted as mapping operators; e.g., the transformation from $\mathcal{L}$ to $\mathcal{B}$ is described as $\Phi_{\mathcal{L} \rightarrow \mathcal{B}}$. However, it is difficult to optimize all four mapping operators simultaneously, so they are optimized one by one. The optimization is conducted for each LF, so different mapping parameters are obtained for different LFs. Therefore, the parameters for the final mapping operator, $\Phi_{\hat{\mathcal{F}} \rightarrow \hat{\mathcal{L}}}$, are transmitted to the receiver along with the HEVC-encoded bit-stream.

\section{2 Mapping operators}

The first mapping operator, $\Phi_{\mathcal{L} \rightarrow \mathcal{B}}$, is defined as

$$
B_{n}(u, v)=\sum_{m} w_{n, m} L_{m}(u, v)
$$

This means that a basis image is synthesized as a weighted average over the input LF images. By imposing the constraints $\sum_{m} w_{n, m}=1$ and $w_{n, m} \geqq 0$, we ensure that the basis images look like the original LF images, with constant brightness across $n$. We also impose a constraint that the first basis image equals the central viewpoint image of the LF, based on our empirical finding that it works well. The weight values $\left(w_{n, m}\right)$ are optimized so that the information in $\mathcal{L}$ is best preserved in $\mathcal{B}$. This optimization is done using a neural network, as described in Section 2.3. Note that the model (Eq. (2)) is similar to the one used by Inagaki et al. for compressive LF acquisition using a coded aperture camera [24], which inspired this study.

Mapping $\Phi_{\mathcal{B} \rightarrow \mathcal{F}}$ is aimed at producing a temporally smooth sequence that is suitable for the video codec. Since only the frame order in $\mathcal{B}$ is changed to produce
$\mathcal{F}$, no information is lost. More specifically, the frame order is optimized to minimize the length, which is defined as

$$
\text { length }=\sum_{n=1}^{N-1} \operatorname{dist}\left(F_{n}(u, v), F_{n-1}(u, v)\right)
$$

where "dist" is a distance measure between two adjacent frames. This is equivalent to the traveling salesman problem, in which the problem is to determine the shortest route for visiting all cities (in our case, frames). Following our previous work [18], we use

$$
\operatorname{dist}\left(F_{n}(u, v), F_{n-1}(u, v)\right)=\sum_{u, v}\left|F_{n}(u, v)-F_{n-1}(u, v)\right|
$$

and the G+2 method of Imaeda et al. [18] to obtain the best possible order.

Mapping $\Phi_{\mathcal{F} \rightarrow \hat{\mathcal{F}}}$ is performed by the HEVC codec, which is regarded as being sufficiently optimized. We consider $\mathcal{F}$ to be a temporal sequence and simply apply HEVC reference software with a random access configuration to obtain a compressed bit-stream. From this bit-stream, we obtain a sequence of decoded frames, $\hat{\mathcal{F}}$.

Mapping operator $\Phi_{\hat{\mathcal{F}} \rightarrow \hat{\mathcal{L}}}$ is defined as

$$
\hat{L}_{m}(u, v)=\sum_{n} \tilde{w}_{m, n} \hat{F}_{n}(u, v)
$$

This mapping corresponds to the inverse of composite mapping $\Phi_{\mathcal{B} \rightarrow \mathcal{F}} \circ \Phi_{\mathcal{L} \rightarrow \mathcal{B}}$, in which the inverse reordering and reconstruction of the LF images are performed in a single step. The parameters $\tilde{w}_{m, n}$ are optimized so that the reconstructed LF $(\hat{\mathcal{L}})$ best approximates the 
original one $(\mathcal{L})$. This optimization is also conducted using a neural network and is described in Section 2.3. Constraint $\tilde{w}_{m, n} \geqq 0$ is imposed to make $\tilde{w}_{m, n}$ sparse. All values for $\tilde{w}_{m, n}$ are transmitted to the receiver side.

The mapping operators obtained for the Bikes LF $(M=193)$ with $N=128$ and $\mathrm{QP}=22$ are shown in Fig. 2. In plots (a),(c) and (d), the weights for $\Phi_{\mathcal{L} \rightarrow \mathcal{B}}\left(w_{n, m}\right.$ as a $N \times M$ matrix $)$, the reordering pattern for $\Phi_{\mathcal{B} \rightarrow \mathcal{F}}$ (as a $N \times N$ permutation matrix), and the weights for $\Phi_{\hat{\mathcal{F}} \rightarrow \hat{\mathcal{L}}}\left(\tilde{w}_{m, n}\right.$ as a $M \times N$ matrix $)$ are visualized. A color map (b) shows the contribution of each input viewpoint to the basis images, where each square corresponds to each viewpoint, and the value (also color-coded for visualization) in each square is obtained as

$$
w_{m}=\sum_{n} w_{m, n}
$$

From this contribution map, we can see the extent of dependency the set of the basis images jointly has on each input viewpoint.

\subsection{Implementation using neural network}

The first and final mapping operators, $\Phi_{\mathcal{L} \rightarrow \mathcal{B}}$ and $\Phi_{\hat{\mathcal{F}} \rightarrow \hat{\mathcal{L}}}$, are implemented using neural networks.

The first mapping $\Phi_{\mathcal{L} \rightarrow \mathcal{B}}$ is optimized on the basis of principal component analysis, which is formulated as

$$
\underset{\Phi_{\mathcal{B} \rightarrow \overline{\mathcal{L}}, \Phi_{\mathcal{L}} \rightarrow \mathcal{B}}}{\arg \min }\left\|\mathcal{L}-\Phi_{\mathcal{B} \rightarrow \overline{\mathcal{L}}} \circ \Phi_{\mathcal{L} \rightarrow \mathcal{B}}(\mathcal{L})\right\|^{2},
$$

where $\Phi_{\mathcal{B} \rightarrow \overline{\mathcal{L}}}$ is a pseudo-inverse operator given as

$$
\bar{L}_{m}(u, v)=\sum_{n} w_{m, n}^{*} B_{n}(u, v) .
$$

By combining this with Eq. (2), we can describe composite mapping $\Phi_{\mathcal{B} \rightarrow \overline{\mathcal{L}}} \circ \Phi_{\mathcal{L} \rightarrow \mathcal{B}}$ as

$$
\bar{L}_{m}(u, v)=\sum_{n} w_{m, n}^{*} \sum_{m} w_{n, m} L_{m}(u, v) .
$$

Focusing on a specific pixel position $((u, v))$, we see that this calculation is implemented as a fully connected neural network with three layers, as shown in the top-left diagram in Fig. 1. The input, intermediate, and output layers in this network have $M, N$, and $M$ nodes, respectively. The input and output are vectors with $M$ elements corresponding to $\left\{L_{m}(u, v) \mid m=0, \ldots, M-1\right\}$ and $\left\{\bar{L}_{m}(u, v) \mid m=0, \ldots, M-1\right\}$, respectively, for a specific $(u, v)$. The intermediate layer corresponds to $\left\{B_{n}(u, v) \mid n=0, \ldots, N-1\right\}$ for the same $(u, v)$. The weight values between the input and intermediate layers correspond to $w_{n, m}$, which are optimized jointly with $w_{m, n}^{*}$ in the training stage. The output from the network is supervised by the original LF images, enforcing
Table 1 PSNR of reconstructed LFs $(\bar{L})$

\begin{tabular}{|r|r|r|r|r|}
\hline$N$ & Bikes & Friends & Flowers & $\begin{array}{c}\text { Stone Pillars } \\
\text { Outside }\end{array}$ \\
\hline 193 & 51.74 & 48.43 & 50.84 & 45.16 \\
\hline 128 & 45.16 & 47.69 & 46.49 & 47.65 \\
\hline 64 & 39.56 & 43.65 & 41.40 & 42.53 \\
\hline 32 & 35.82 & 40.58 & 37.67 & 38.83 \\
\hline
\end{tabular}

that $\bar{L}_{m} \simeq L_{m}$ for all $m$, in a least-squares sense. We use all the pixels in the LF as the training dataset, which means that the obtained weight values $\left(w_{n, m}\right)$ are optimized for all the pixels but only for the target LF.

In Table 1, we present the quality of a reconstructed LF $(\bar{L})$ to show how much information is preserved in the basis images without the HEVC coding. We used the same four LFs as those used in Section $3(M=193)$. We set $N$ to $193,128,64,32$. Here, the peak signal-toratio (PSNR) is calculated using the mean squared error of all 193 images. We can see the reconstruction quality depends on the number of basis images $N$; Generally, $N$ should be larger for higher reconstruction quality.

The final mapping $\left(\Phi_{\hat{\mathcal{F}} \rightarrow \hat{\mathcal{L}}}\right)$ is also optimized:

$$
\underset{\Phi_{\hat{\mathcal{F}} \rightarrow \hat{\mathcal{L}}}}{\arg \min }\left\|\mathcal{L}-\Phi_{\hat{\mathcal{F}} \rightarrow \hat{\mathcal{L}}}(\hat{\mathcal{F}})\right\|^{2} .
$$

In a manner similar to the one described above, Eq. (5) is implemented as a fully connected neural network for individual pixels, as shown in the topright diagram in Fig. 1. The network has two layers, the input and output layers, which have $N$ and $M$ nodes, respectively. The input consists of the decoded frames $\left(\left\{\hat{F}_{n}(u, v) \mid m=0, \ldots, N-1\right\}\right)$ for a specific $(u, v)$, and the output consists of the reconstructed $\operatorname{LF}\left(\left\{\hat{L}_{m}(u, v) \mid m=0, \ldots, M-1\right\}\right)$ for the same pixel. The weights between the layers correspond to $\tilde{w}_{m, n}$. The output from the network is supervised by the original LF images, enforcing that $\hat{L}_{m} \simeq L_{m}$ for all $m$ in a least-squares sense. We use all the pixels in the target LF as the training dataset. The training is conducted for each configuration (e.g., the quantization parameter (QP) value) of the HEVC codec because the configuration greatly affects the decoded frames $(\hat{\mathcal{F}})$, which are the network input. All the parameters $\left(\tilde{w}_{m, n}\right)$ are optimized on the sender side and transmitted to the receiver side along with the HEVC-encoded bit-stream.

The neural networks were implemented using Keras version 2.2.4 and the Adam Optimizer. The size of mini-batch was set to 16,384 . The number of epochs was set to 3000 and 500 for $\Phi_{\mathcal{L} \rightarrow \mathcal{F}}$ and $\Phi_{\hat{\mathcal{F}} \rightarrow \hat{\mathcal{L}}}$, respectively. 


\section{Evaluation}

We used grayscale LFs from the EPFL light-field data set [25], following the guidelines of the ICME 2016 Grand Challenge [26]. Each LF had $15 \times 15$ images with a resolution of $625 \times 434$ pixels. The viewpoints near the corners were removed because they were noisy and distorted, so the number of viewpoints used $(M)$ was 193. HEVC reference software (version 16.2) [23] was used to encode and decode the intermediate video in YUV400 format. Random access was used for the inter-frame prediction structure, resulting in excellent rate-distortion performance. The PSNR averaged over all 193 images was used as the quality metric. The bitrate (bits per pixel: BPP) was calculated using

$$
\mathrm{BPP}=\frac{R_{s}+R_{w}}{625 \times 434 \times 193},
$$

where $R_{s}$ and $R_{w}$ represent the size (in bits) for the HEVC bit-stream and the weight parameters $\tilde{w}_{m, n}$, respectively. The weight parameters were rounded to 16 bit and compressed in LZMA format. Our experimental environment is summarized in Table 2.

The computation time for encoding an LF (we used Bikes LF) with $N=193,128,64,32$ and $\mathrm{QP}=22$ is shown in Table 3 . Table 4 shows the breakdown between $R_{s}$ and $R_{w}$ for various configurations on Bikes LF. We can see that $R_{w}$ has only a limited ratio in the total bit length.

The performance of our method on four LFs is shown in Fig. 3(a). We tested different values for $N(32,64$, 128, and 193). In terms of the overall performance, $N=128$ is the best choice among them. However, as shown in the close-ups, using $N=32$ or 64 led to better rate-distortion performance in the low bit-rate. We also presented several contribution maps for the same LFs in Fig. 3(b)-(d). From these maps, we can see that depending on the target LF and the number of basis images, the extent of contribution from each input viewpoint is significantly different.

We also present two ablation studies. First, we nullified the frame reordering $\left(\Phi_{\mathcal{B} \rightarrow \mathcal{F}}\right.$ in Fig. 1) and show some results in Fig. 4. From these results, we can see that the frame reordering contributes to the ratedistortion performance, in particular for a large value of $N$. Second, we removed the process of synthesizing basis images $\left(\Phi_{\mathcal{L} \rightarrow \mathcal{B}}\right.$ in Fig. 1). In the ablated cases, we randomly or quasi-evenly selected the input views and used them as the basis images. Shown on the top
Table 2 Experimental environment

\begin{tabular}{|l|l|}
\hline OS & Linux ubuntu16.04 LTS \\
\hline PC & HP EliteDesk 800 G2 SFF \\
\hline Main memory & 40GB \\
\hline CPU & Intel Core i7-8700 CPU \\
\hline GPU & NVIDIA GeForce GTX1070 Ti \\
\hline
\end{tabular}

Table 3 Encoding time [sec]

\begin{tabular}{|c|r|r|r|r|r|}
\hline$N$ & $\Phi_{\mathcal{L} \rightarrow \mathcal{B}}$ & $\Phi_{\mathcal{B} \rightarrow \mathcal{F}}$ & $\Phi_{\mathcal{F} \rightarrow \hat{\mathcal{F}}}$ & $\Phi_{\hat{\mathcal{F}} \rightarrow \hat{\mathcal{L}}}$ & total time \\
\hline \hline 193 & $4.25 \times 10^{3}$ & 7.94 & $5.84 \times 10^{2}$ & $6.82 \times 10^{2}$ & $5.52 \times 10^{3}$ \\
\hline 128 & $4.08 \times 10^{3}$ & 2.85 & $4.23 \times 10^{2}$ & $5.52 \times 10^{2}$ & $5.06 \times 10^{3}$ \\
\hline 64 & $3.90 \times 10^{3}$ & 0.69 & $2.31 \times 10^{2}$ & $4.28 \times 10^{2}$ & $4.56 \times 10^{3}$ \\
\hline 32 & $3.89 \times 10^{3}$ & 0.23 & $1.18 \times 10^{2}$ & $3.81 \times 10^{2}$ & $4.39 \times 10^{3}$ \\
\hline
\end{tabular}

Table 4 Breakdown between $R_{s}$ and $R_{u}$

\begin{tabular}{|c|c|r|r|r|r|}
\hline$N$ & QP & PSNR $[\mathrm{dB}]$ & $\mathrm{BPP}\left(\times 10^{-3}\right)$ & $R_{s}[\mathrm{kbit}]$ & $R_{w}[\mathrm{kbit}]$ \\
\hline \hline \multirow{4}{*}{128} & 22 & 38.92 & 129.15 & $6,737,192$ & 23,944 \\
\cline { 2 - 6 } & 27 & 36.22 & 47.46 & $2,462,160$ & 22,584 \\
\cline { 2 - 6 } & 32 & 33.88 & 20.34 & $1,041,440$ & 23,520 \\
\cline { 2 - 6 } & 37 & 31.56 & 9.52 & 473,656 & 24,704 \\
\hline \multirow{4}{*}{64} & 22 & 37.15 & 77.76 & $4,054,752$ & 16,184 \\
\cline { 2 - 6 } & 27 & 35.13 & 29.95 & $1,551,168$ & 16,528 \\
\cline { 2 - 6 } & 32 & 33.15 & 13.50 & 689,840 & 16,768 \\
\cline { 2 - 6 } & 37 & 31.06 & 6.61 & 326,536 & 19,584 \\
\hline \multirow{4}{*}{32} & 22 & 34.91 & 46.86 & $2,440,240$ & 12,856 \\
\cline { 2 - 6 } & 27 & 33.61 & 19.42 & $1,003,576$ & 13,040 \\
\cline { 2 - 6 } & 32 & 32.17 & 9.33 & 475,008 & 13,584 \\
\cline { 2 - 6 } & 37 & 30.47 & 4.73 & 233,552 & 14,216 \\
\hline
\end{tabular}

and middle of Fig. 5 are examples of randomly selected views and quasi-evenly spaced views with different $N$ $(32,64$, and 128). Here, each square corresponds to the location of each input viewpoint, and the number and color of each square indicate the frame order in which the selected views are aligned in a temporal sequence (the frame order was optimized using the same method as ours). The final mapping operator $\Phi_{\hat{\mathcal{F}} \rightarrow \hat{\mathcal{L}}}$ was optimized for each case in the same manner as ours. The rate-distortion performance is shown in the graphs at the bottom of Fig. 5, where we performed random selection for three times. We can see that our scheme of synthesizing basis images lead to better performance than random selection, in particular with a small number of $N$. Using quasi-evenly spaced viewpoints resulted in almost the same performance as our scheme for $N=128$ and 64. However, the advantage of our scheme over the quasi-even one became obvious for $N=32$.

We also compared our method against other HEVCbased methods. We tested four representative frame orders (Raster [12], Circular [12,13], LF-CAE [17] and LFTSP $(G+2)[18])$, as shown in matrices (a)-(d) in Fig. 6. Note that the frame orders obtained by LF-CAE and LF-TSP $(\mathrm{G}+2)$ were different for different LFs. Following the original implementation [17], LF-CAE was implemented with Low delay B for inter-frame predic- 

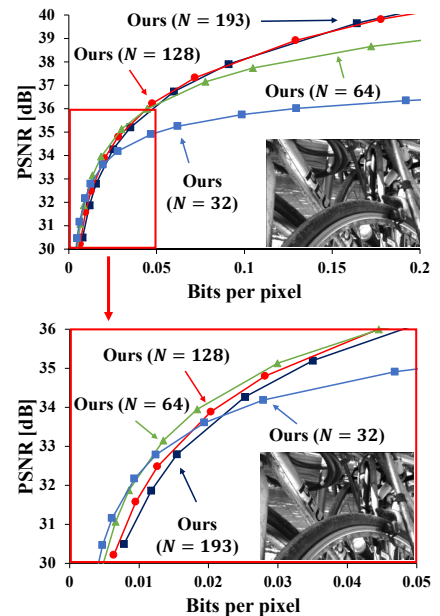

(a) (top) Overall rate-distortion perform
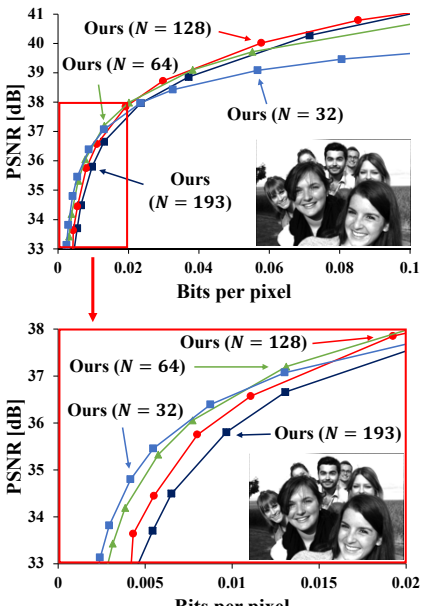
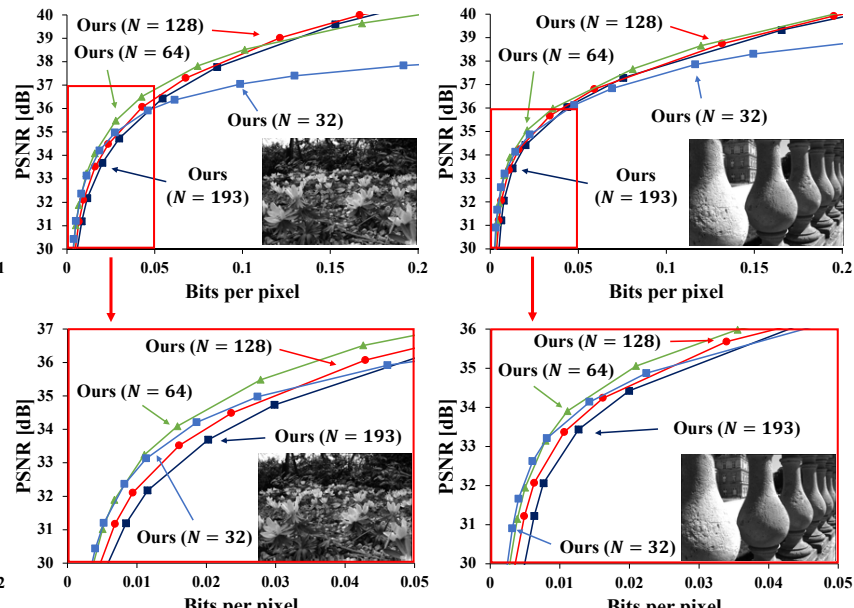


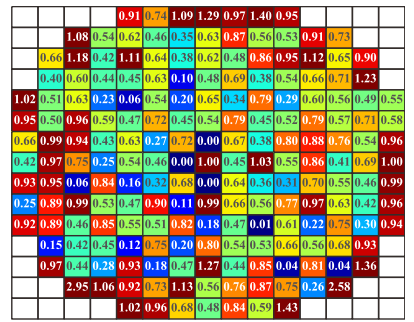

(b) Contribution maps $(N=128)$



(c) Contribution maps $(N=64)$

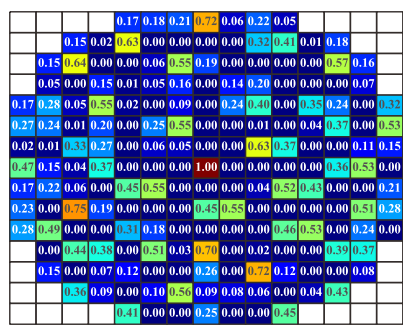

(d) Contribution maps $(N=32)$
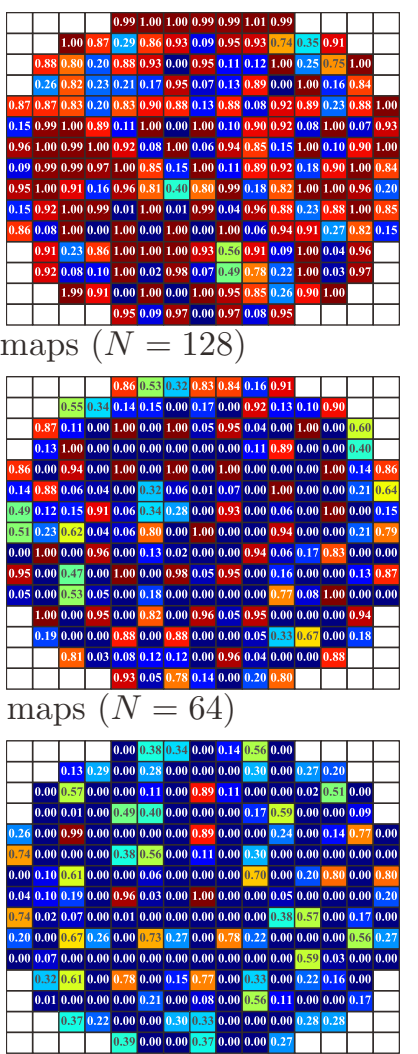


Fig. 3 Rate-Distortion performance and contribution maps of our method on four different LFs (from left to right, Bikes, Friends, Flowers, and Stone Pillars Outside)

tion structure* ${ }^{*}$ The number of basis images $N$ was set to 128 for our method. As shown in the rate-distortion curves in (e)-(h), our method had better rate-distortion performance than the other methods. It should be noted that our method also used LF-TSP $(\mathrm{G}+2)$ [18] for reordering the basis images, so the performance difference between our method and LF-TSP $(G+2)$ was due solely to the use of basis images. Table. 5 shows Bjøntegaard delta rates (BD-rates) [27], showing bit-

\footnotetext{
* Using Random access structure instead of Low delay B slightly improved the performance of LF-CAE, but it was still much inferior to LF-TSP $(\mathrm{G}+2)$ as shown in [18].
}

rate reduction ratios from the reference at the same quality. The reference was set to the Raster order. We can see that our method achieved the best performance with $25.34 \%$ rate reduction in average compared to the reference case.

\section{Conclusion}

We proposed an HEVC-based light-field coding method using basis images and frame reordering. Our method first condenses the original LF into a set of basis images and then reorders them to create a temporally smooth video sequence that is HEVC codec friendly. 

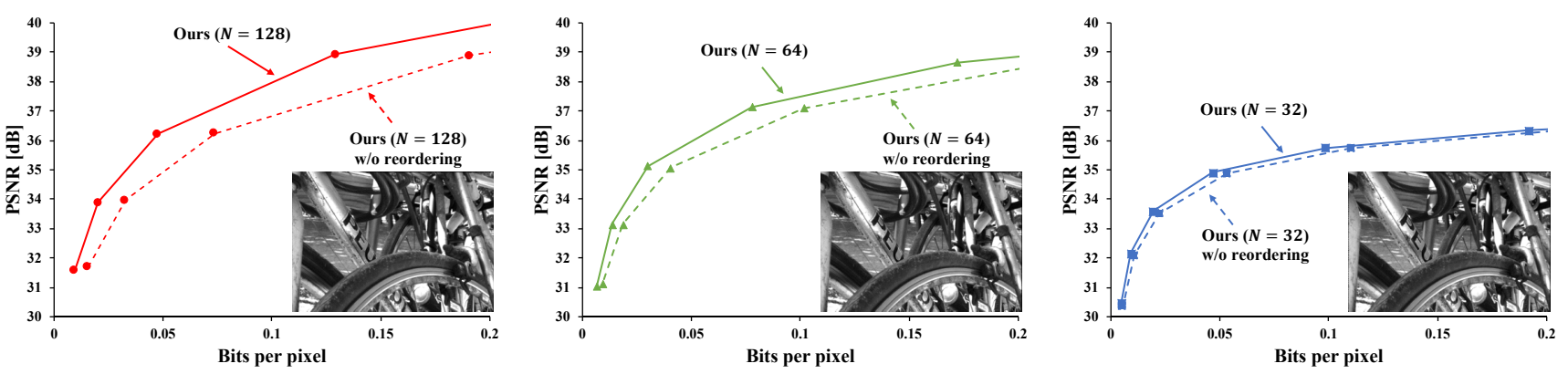

Fig. 4 Ablation study: frame reordering $\left(\Phi_{\mathcal{B} \rightarrow \mathcal{F}}\right)$ was ablated.


(Random) Randomly selected input viewpoints and their order
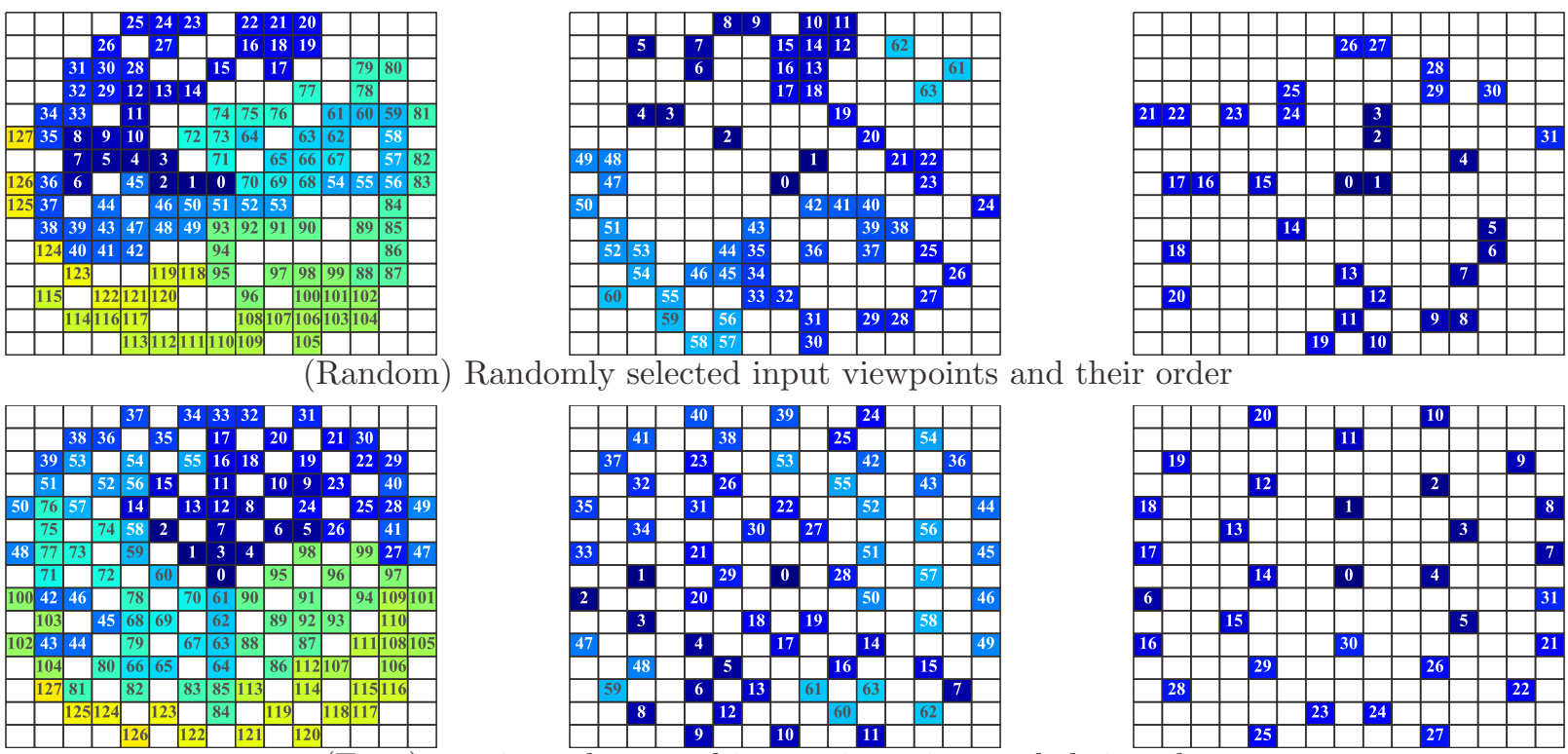

(Even) quasi-evenly spaced input viewpoints and their order

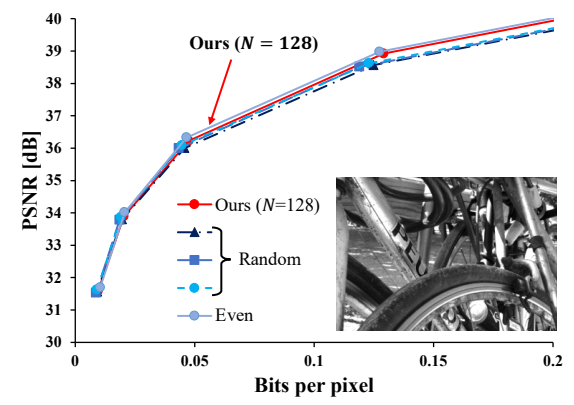

(a) $N=128$

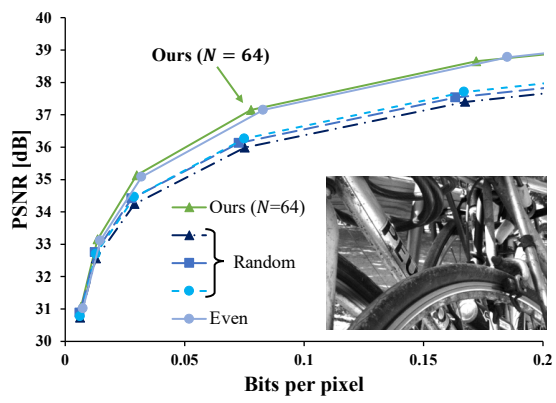

(b) $N=64$

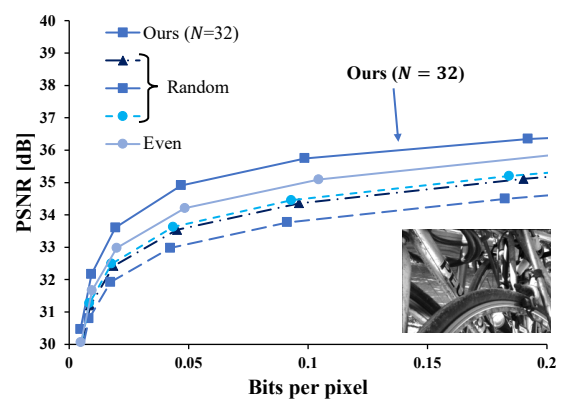

(c) $N=32$

Fig. 5 Ablation study: synthesis of basis images $\left(\Phi_{\mathcal{L} \rightarrow \mathcal{B}}\right)$ was ablated. (top) Randomly selected input viewpoints and their order, (middle) quasi-evenly spaced input viewpoints and their order,

(bottom) rate-distortion performance.

Experimental results show that the design choices of our method were effective and our method has promising performance compared to previous works.

Future work will take several directions. First, we will apply our method to light-field datasets other than ones taken using Lytro cameras, which may result in a different setting for the number of basis images. Instead of the current video coding standard (HEVC), the upcoming video coding standard called Versatile Video Coding (VVC) [28] can also be combined with our method. We also intend to investigate other coding methods including 3D HEVC [7,10], and learned video coding methods [19-22] to clarify the strength of each method and to evaluate the potential synergy with our method. Finally, we hypothesize that generating basis images is also effective for other applications such as coding of high-speed videos.

\section{References}

1) E. H. Adelson, J. R. Bergen, et al.: "The plenoptic function and the elements of early vision", Computational Models of Visual Processing, pp. 3-20, (1991).

2) S. J. Gortler, R. Grzeszczuk, R. Szeliski, and M. F. Cohen: "The lumigraph", Proceedings of the 23rd annual conference on Com- 


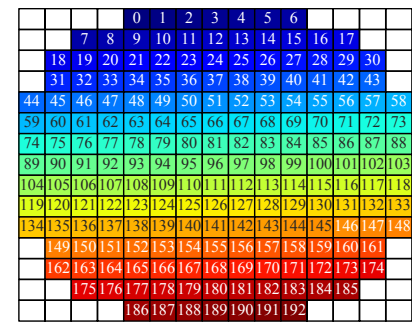

(a) Raster

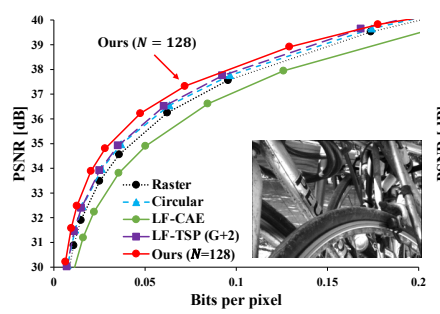

(e) Bikes

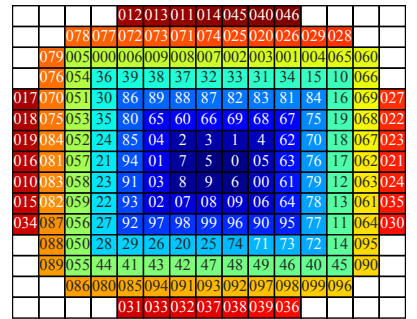

(b) Circular

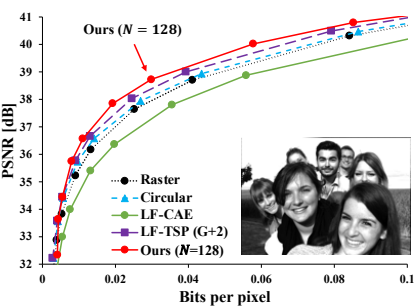

(f) Friends

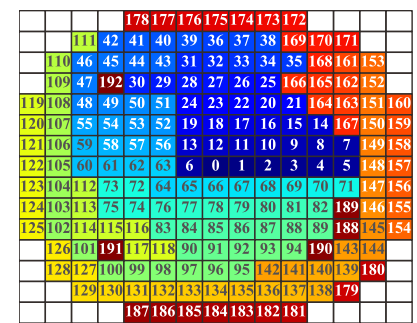

(c) LF-CAE for Bikes

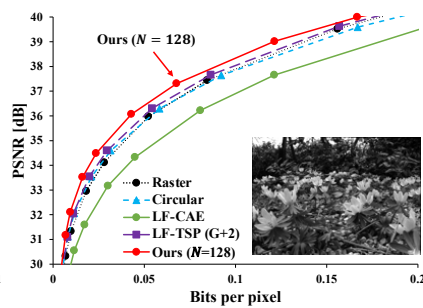

(g) Flowers

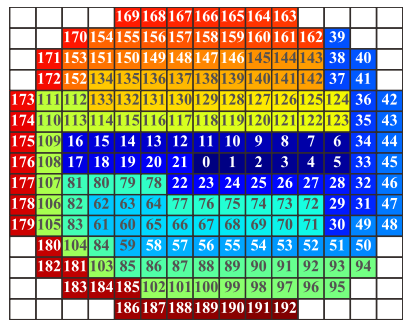

(d) LF-TSP $(\mathrm{G}+2)$ for Bikes

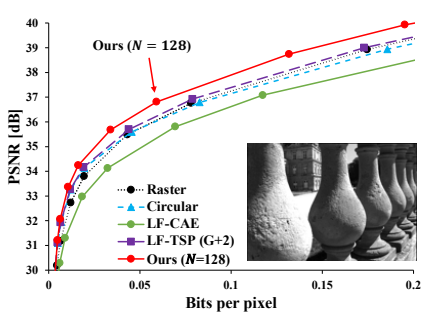

(h) Stone Pillars Outside

Fig. 6 Comparison of our method against previous methods. (a)-(d) Frame orders in previous methods. (e)-(h) Rate-distortion curves for four LFs (Bikes, Friends, Flowers, and Stone Pillars Outside).

Table 5 BD-rate

\begin{tabular}{|c|r|r|r|r|r|}
\hline method & Bataset & Friends & Flowers & Stone Pillars Outside & AVERAGE \\
\hline Circular & -8.72 & -9.31 & -2.62 & -7.55 & -7.05 \\
\hline LF-CAE & 23.63 & 44.82 & 31.94 & 35.37 & 33.94 \\
\hline LF-TSP $(\mathrm{G}+2)$ & -11.59 & -17.98 & -8.55 & -13.28 & -12.85 \\
\hline Ours $(N=128)$ & $\mathbf{- 2 4 . 3 0}$ & $\mathbf{- 2 6 . 5 0}$ & $\mathbf{- 2 2 . 5 1}$ & $\mathbf{- 2 8 . 0 4}$ & $\mathbf{- 2 5 . 3 4}$ \\
\hline
\end{tabular}

puter graphics and interactive techniques, pp. 43-54, (1996).

3) S. Wanner and B. Goldluecke: "Variational light field analysis for disparity estimation and super-resolution", IEEE transactions on pattern analysis and machine intelligence, vol. 36, no. 3, pp. 606-619, (2014).

4) T. Saito, Y. Kobayashi, K. Takahashi, and T. Fujii: "Displaying real-world light fields with stacked multiplicative layers: requirement and data conversion for input multiview images", Journal of Display Technology, vol. 12, no. 11, pp. 1290-1300, (2016).

5) E. H. Adelson and J. Y. A. Wang: "Single lens stereo with a plenoptic camera", IEEE Transactions on Pattern Analysis and Machine Intelligence, vol. 14, no. 2, pp. 99-106, (Feb. 1992).

6) R. Ng, M. Levoy, M. Brédif, G. Duval, M. Horowitz, P. Hanrahan, et al.: "Light field photography with a hand-held plenoptic camera", Computer Science Technical Report CSTR, vol. 2, no. 11, pp. 1-11, (2005).

7) G. Tech, Y. Chen, K. Müller, J. R. Ohm, A. Vetro, and Y. K. Wang: "Overview of the multiview and 3D extensions of high efficiency video coding", IEEE Transactions on Circuits and Systems for Video Technology, vol. 26, no. 1, pp. 35-49, (2016).

8) D. Liu, L. Wang, L. Li, Z. Xiong, F. Wu, and W. Zeng: "Pseudo-sequence-based light field image compression", 2016 IEEE International Conference on Multimedia \& Expo Workshops (ICMEW), pp. 1-4, (2016).

9) X. Jiang, M. Le Pendu, R. A. Farrugia, and C. Guillemot: "Light field compression with homography-based low-rank approximation", IEEE Journal of Selected Topics in Signal Processing, vol. 11 , no. 7 , pp. 1132-1145, (2017).

10) W. Ahmad, R. Olsson, and M. Sjöström: "Interpreting plenoptic images as multi-view sequences for improved compression", 2017 IEEE International Conference on Image Processing (ICIP), pp. 4557-4561, (Sep. 2017).

11) K. Komatsu, K. Takahashi, and T. Fujii: "Scalable light field coding using weighted binary images", 2018 IEEE International Conference on Image Processing (ICIP), pp. 903-907, (Oct. 2018).

12) F. Dai, J. Zhang, Y. Ma, and Y. Zhang: "Lenselet image compression scheme based on subaperture images streaming", 2015 IEEE International Conference on Image Processing (ICIP), pp. 4733-4737, (Sep. 2015)

13) A. Vieira, H. Duarte, C. Perra, L. Tavora, and P. Assuncao:
"Data formats for high efficiency coding of Lytro-illum light fields", 2015 International Conference on Image Processing Theory, Tools and Applications (IPTA), pp. 494-497, (Nov. 2015).

14) S. Zhao, Z. Chen, K. Yang, and H. Huang: "Light field image coding with hybrid scan order", 2016 Visual Communications and Image Processing (VCIP), pp. 1-4, (Nov. 2016).

15) "ITU-T Recommendation H.265 and ISO/IEC 23008-2: High efficiency video coding", (Feb. 2018).

16) G. J. Sullivan, J. R. Ohm, W. J. Han, T. Wiegand, et al.: "Overview of the high efficiency video coding (HEVC) standard", IEEE Transactions on circuits and systems for video technology, vol. 22 , no. 12 , pp. 1649-1668, (2012).

17) R. Conceição, M. Porto, B. Zatt, and L. Agostini: "LF-CAE: Context-adaptive encoding for lenslet light fields using HEVC", 2018 IEEE International Conference on Image Processing (ICIP), pp. 3174-3178, (2018).

18) K. Imaeda, K. Isechi, K. Takahashi, T. Fujii, S. Takamura, Y. Bandoh, T. Miyazawa, and A. Shimizu: "LF-TSP: Traveling salesman problem for HEVC-based light-field coding", 2019 Visual Communications and Image Processing (VCIP), (2019).

19) J. Han, S. Lombardo, C. Schroers, and S. Mandt: "Deep probabilistic video compression", arXiv preprint arXiv:1810.02845, (2018).

20) O. Rippel, S. Nair, C. Lew, S. Branson, A. G. Anderson, and L. Bourdev: "Learned video compression", 2019 IEEE International Conference on Computer Vision (ICCV), pp. 3454-3463, (Oct. 2019).

21) C. Wu, N. Singhal, and P. Krahenbuhl: "Video compression through image interpolation", The European Conference on Computer Vision (ECCV), pp. 416-431, (Sep. 2018).

22) A. Habibian, T. V. Rozendaal, J. M. Tomczak, and T. S. Cohen: "Video compression with rate-distortion autoencoders", 2019 IEEE International Conference on Computer Vision (ICCV), pp. 7033-7042, (Oct. 2019).

23) K. McCann, C. Rosewarne, B. Bross, M. Naccari, K. Sharman, and G. Sullivan: "High efficiency video coding (HEVC) test model 16 (HM 16) improved encoder description", ISO/IEC JTC1/SC29/WG11 N14970, (2014).

24) Y. Inagaki, Y. Kobayashi, K. Takahashi, T. Fujii, and H. Nagahara: "Learning to capture light fields through a coded aperture camera", The European Conference on Computer Vision 
(ECCV), pp. 418-434, (Sep. 2018).

25) M. Rerabek and T. Ebrahimi: "New light field image dataset", 8th International Conference on Quality of Multimedia Experience(QoMEX), pp. 1-2, (2016).

26) M. Rerabek, T. Bruylants, T. Ebrahimi, F. Pereira, and P. Schelkens: "ICME 2016 grand challenge: Light-field image compression", Call for proposals and evaluation procedure, (2016).

27) G. Bjøntegaard: "Calculation of average PSNR differences between RD-curves", Proceedings of the ITU-T Video Coding Experts Group (VCEG) Thirteenth Meeting, (2001).

28) K. H. Seung C. Jianle, Y. Yan: "Algorithm description for versatile video coding and test model 7 (VTM 7)", ISO/IEC JTC 1/SC 29/WG 11 N18874, (2019).



Kota Imaeda received a B.E. degree in electrical engineering from Nagoya University, Nagoya, Japan, in 2019, where he is currently working toward a graduate degree in electrical engineering and computer science. His research project includes light field compression.

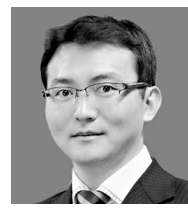

Keita Takahashi received B.E., M.S., and Ph.D. degrees in information and communication engineering from the University of Tokyo, Tokyo, Japan, in 2001, 2003, and 2006. He was a Project Assistant Professor at the University of Tokyo from 2006 to 2011 and was an Assistant Professor at the University of Electro-Communications from 2011 to 2013. He is currently an Associate Professor at the Graduate School of Engineering, Nagoya University, Nagoya, Japan. His research interests include computational photography, image-based rendering, and 3-D displays.

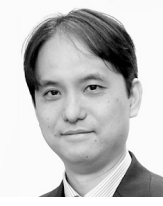

Toshiaki Fujii received B.E., M.E., and Dr.E. degrees in electrical engineering from the University of Tokyo, Tokyo, Japan, in 1990, 1992, and 1995. In 1995, he joined the Graduate School of Engineering, Nagoya University, where currently he is a Professor. From 2008 to 2010, he was with the Graduate School of Science and Engineering, Tokyo Institute of Technology. His current research interests include multidimensional signal processing, multi-camera systems, multi-view video coding and transmission, free-viewpoint television, and their applications.

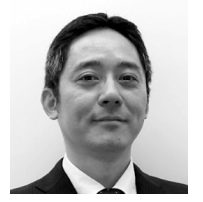

Yukihiro Bandoh received the B.E., M.E. and Ph.D. degrees from Kyushu University, Japan, in 1996, 1998 and 2002, respectively. He granted JSPS Research Fellowship for Young Scientists from 2000 to 2002. In 2002, he joined Nippon Telegraph and Telephone (NTT) Corporation, where he has been engaged in research on efficient video coding for high realistic communication. He is currently a Distinguished Engineer at NTT Media intelligence Laboratories. His current research interests include efficient video coding and sparse modeling.



Seishi Takamura received B.E., M.E., and $\mathrm{Ph} . \mathrm{D}$. degrees in electrical engineering from the University of Tokyo, Tokyo, Japan, in 1991, 1993, and 1996. In 1996, he joined NTT Human Interface Laboratories, NTT Corporation, where currently he is a Senior Distinguished Engineer. From 2005 to 2006, he was a visiting scientist at Stanford University, CA, USA. His current research interests include efficient video coding and multi-modal signal information processing.

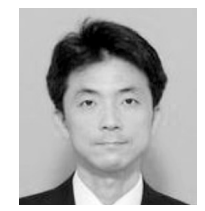

Hideaki Kimata eceived the B.E. an M.E. degrees in applied physics, and the Ph.D degree in electrical engineering respectively from Nagoya University, Nagoya, Japan, in 1993, 1995 and 2006. He joined Nippon Telegraph and Telephone Corporation (NTT) in 1995, and has been involved in the research and development of video coding, realistic communication, computer vision, and video recognition based on machine learning (deep learning). He is currently a Senior Research Engineer and also the Supervisor at NTT Media Intelligence Laboratories. He is a Chair of Technical Committee on Image Engineering of the Institute of Electronics, Information and Communication Engineers of Japan. 